\title{
Informational and neural adaptation curves are asynchronous
}

\author{
LAWRENCE M. WARD \\ University of British Columbia, Vancouver, British Columbia
}

\begin{abstract}
Norwich's entropy theory of perception (plus a few additional assumptions) suggests the existence of an "informational adaptation curve" (change in stimulus equivocation with stimulus duration) for suprathreshold prothetic stimuli that is synchronous with the "neural adaptation curve" (change in firing rate with stimulus duration) observed for sensory neurons. Five experiments are reported in which informational adaptation curves were measured for auditory and visual stimuli by having subjects make absolute identifications of suprathreshold sound or light intensities of various durations. Information transmissions for the shortest duration stimuli (1 and $5 \mathrm{msec}$, respectively, for light and sound) were surprisingly large (small equivocations), indicating that intensity information is acquired very rapidly by the whole organism. The equations of entropy theory were fitted to adaptation data for peripheral sensory neurons (spiral ganglion cells and retinal ganglion cells) and were compared to the informational adaptation curves. It was found that informational adaptation occurred more rapidly than neural adaptation. That is, the two types of adaptation process are asynchronous. However, for both audition and vision, the total amount of information mediated by the adaptation process (channel capacity) was about the same for both types of processes (2.03 bits vs. 2.1 bits per stimulus for sound intensity; 1.3 vs. 2.0 bits per stimulus for light intensity). Faster acquisition could be accomplished in the whole organism through convergent neural circuits that increase sampling rate by pooling the samples taken by individual receptor systems.
\end{abstract}

It is nearly dogma in the study of sensation and perception that the duration of a stimulus affects the amount of information transmitted from it to an observer. It is obvious in studies of more complex perceptions that the stimulus duration limits performance, and stimulus duration is often manipulated for that very purpose (see, e.g., Paquet \& Merikle, 1984). Moreover, absolute threshold (see, e.g., Hughes, 1946) and differential threshold (see, e.g., Florentine, 1986) vary with stimulus duration for constantintensity stimuli. Information transmission for absolute identification of suprathreshold stimuli has also been thought to vary with stimulus duration (cf. Garner, 1962), although there appear to have been few studies in which changes in stimulus duration were studied in isolation. For example, Lockhead (1966) varied both stimulus duration (200 vs. $8 \mathrm{msec}$ ) and luminance (no filter vs. $1 \mathrm{log}$ unit neutral density filter) in absolute identification of line

This research was supported by a grant from the Natural Sciences and Engineering Research Council of Canada. I am grateful to Ken Norwich for many discussions regarding this work and for his insightful comments on this paper; for bringing the papers by Yates, Robertson, and Johnstone (1985) and Cleland and Enroth-Cugell (1968) to my attention; for supplying the simplex optimization algorithm; and for providing access to his own unpublished work. I thank Odie Geiger, Steven Lindsay, and Kelly Davidson for help with running subjects, and Shuji Mori for help with the setup for the visual stimuli. Requests for reprints should be sent to Lawrence M. Ward, Department of Psychology, University of British Columbia, 2136 West Mall, Vancouver, BC V6T 174 , Canada. lengths to decrease information transmission from 1.07 bits to 0.19 bit. This procedure is typical (cf. Mori, 1989).

Three studies done for other reasons did contain conditions in which absolute identifications of stimuli of various durations were made without serious confounding by other variables. First, Garner and Creelman (1964) studied the effects of different kinds of redundancy on absolute identifications of visual stimuli that varied in hue or size. Stimulus duration itself was considered to be a kind of redundancy, with two or more contiguous time periods providing correlated stimulus exposures. The other kind of redundancy studied was the addition of stimulus variation on a correlated dimension (size or hue alone versus size and hue varying together). This early study resulted in a significant redundancy gain from combining size and hue variation (about $0.6-1.0$ bit above the baseline unidimensional conditions) but very little gain (less than 0.1 bit) from increasing stimulus duration from 40 to $100 \mathrm{msec}$. Garner and Creelman suggested that absolute identification imposes limitations on performance beyond those of data acqusition, which are reflected in the effect of duration on detection and discrimination. I will return to this point in the discussion. For the present, it will suffice to point out that hue is not an "intensity" (prothetic) stimulus dimension, and that size is a somewhat unusual one (e.g., its coding is probably based on substitution of activity of neurons with variously sized receptive fields rather than on differences in the amount of activity in a particular set of neurons), so Garner and Creelman's conclusion may be limited in generality. Ex- 
periments with more prototypical prothetic continua could give somewhat different results.

Second, Tulving and Lindsay (1967) tested Broadbent's (1958) sequential processing hypothesis (that a person cannot effectively divide attention between two stimuli). They compared information transmission when visual or auditory stimuli were presented singly with information transmission when stimuli from both modalities were presented simultaneously, for stimuli of both short $(20-$ or $50-\mathrm{msec})$ and long (2-sec) durations. Stimulus duration affected information transmission for single-modality presentations of sound and light intensity, sound frequency, and size of visual stimuli (circles), but only by a small amount (about 0.25 bit) and somewhat inconsistently (little effect for high intensities).

Finally, Egeth and Pachella (1969) attempted to explain why, although total information transmitted by multidimensional stimuli increases as the number of dimensions increases, information transmitted about each component dimension decreases. Among other things, they showed that duration affected information transmission on the dimensions of dot position $(0.75 \mathrm{bit})$, hue $(0.30 \mathrm{bit})$, eccentricity ( $0.04 \mathrm{bit})$, and size $(0.41 \mathrm{bit})$ of ellipses of colored paper. Surprisingly, in each of these studies, information transmission for the shortest duration stimuli $(20,50,100$, or $125 \mathrm{msec})$ was rather high (usually greater than 1.5 bits), indicating the possibility that effective stimulus duration for even short-duration stimuli may be increased by the availability of a preperceptual memory. The significant (though small) effect of duration for low-intensity stimuli found by Tulving and Lindsay (1967), for which such a preperceptual memory would be weakest, seems to confirm this speculation. However, in the latter study, information transmission for the lowintensity stimuli at the $50-\mathrm{msec}$ stimulus duration averaged 1.62 bits for sound frequency and 2.51 bits for circle size. Either preperceptual memory was still substantial for these weak stimuli, or there is some other reason why such brief stimuli can be identified nearly as well as much longer duration stimuli.

As is often the case with dogma, the belief in a direct relationship between stimulus duration and information transmission has remained tacit for some time, and the reasons for apparent failures of the relationship have not been explored. However, Norwich (1981) has provided a theoretical reason to expect such a relationship for $a b-$ solute identifications of stimuli varying only in intensity and has predicted the general form of it. It should be understood at the outset that Norwich's theory is a theory of laws of information balance or conservation of information. It is not meant to replace laws of sensory, perceptual, or judgmental mechanisms but to complement them. Thus, although some very general assumptions about "mechanism" are made-Norwich, for example, posits receptors that "sample" stimulus energies-these mechanisms are informational and may be implemented in very different ways in different receptor systems. More will be said about this as the aspects of the theory applicable to the effects of stimulus duration on the amount of information gained from that stimulus are developed in what follows. It should be clear, however, that for such a question, a theory of information transfer from environment to organism would be highly relevant.

The entropy theory of perception asserts that we can perceive only when we are uncertain (see, e.g., Norwich, 1977), and that the level of uncertainty at any time with respect to some stimulus can be measured with Shannon's (1948) measure of uncertainty (or entropy), the same measure that has been used in the version of "information theory" that has been applied to performance in absolute identification experiments. Norwich's theory was originally cast in terms of the physiology of sensory "receptorneuron units" (receptor plus primary afferent neuron). A typical receptor-neuron unit is viewed as initially "uncertain" about the mean intensity of a physical stimulus impinging on it. As time passes, it repeatedly samples the flux of energy at the receptor surface (which we know from quantum physics is best viewed at that scale in the form of discrete "chunks" or "quanta"; cf. Hecht, Schlaer, \& Pirenne, 1942), thereby gaining information about the stimulus and reducing its initial uncertainty. The theory interprets the neural activity output by the receptorneuron unit as a "read-out" of its current state of "uncertainty" (or entropy in the information theory sense) vis à vis the mean intensity of a physical stimulus. (This should not be seen as an alternative to the traditional view that the receptor's output is a code for stimulus intensity, but as a different way of interpreting that output for a different purpose - that of deriving laws of information balance.) The simplest possible relationship is assumed in the absence of any definite information about the "true" relationship:

$$
F=k H_{s},
$$

where $F$ represents frequency of action potentials produced by a primary afferent neuron, $H_{s}$ represents the entropy of the receptor-neuron unit, and $k$ is a constant. This is similar to the very useful relationship assumed in physics to hold between thermodynamic entropy, $S$, and Boltzmann's $H$ measure of "uncertainty of microstate," $S=-k H$, where $k$ is Boltzmann's constant (Tolman, 1938). Expressing uncertainty (entropy) in terms of Shannon's information theory applied to continuous probability density functions (which have as parameters the variability of the stimulus intensity about its mean, $\sigma^{2}$, and that of the inevitable noise in the sampling process about its mean, $N^{2}$ ), we have the following expression for the entropy of the receptor-neuron unit (in bits; Norwich, 1981):

$$
H_{s}=[1 /(2 \cdot \ln 2)] \cdot \ln \left(1+\sigma^{2} / N^{2}\right) .
$$

Since the sampling distribution of the mean has variance 
$\sigma^{2} / n$, where $n$, the number of samples taken, is a function of time, $n=\gamma \tau$, and taking $B=\sigma^{2} / \gamma N^{2}$, Equation 2 can be written as

$$
H_{s}=[1 /(2 \cdot \ln 2)] \cdot \ln (1+B / \tau) .
$$

Notice that we are assuming that the noise variance, $N^{2}$, is constant over time, since it is added by the sampling process rather than sampled by it (similarly to the error term in the general linear model). Inserting Equation 3 into Equation 1 yields an expression for the neural activity of a receptor-neuron unit in response to a stimulus of constant intensity:

$$
F=[k /(2 \cdot \ln 2)] \cdot \ln (1+B / \tau) .
$$

Equation 4 shows that the theory predicts that as time since stimulus onset $(\tau)$ increases, neural response to the stimulus decreases, which is equivalent to neural adaptation to the stimulus, a well-known property of sensory neurons (the law of adaptation). Again, this interpretation should not be seen to be inconsistent with the more traditional interpretation of adaptation as, for example, neural fatigue, but rather to interpret the adaptation in a different way for the purpose of understanding information transfer from environment to receptor system. With a few additional assumptions, especially that for most physical continua the variance, $\sigma^{2}$, is a power function of the stimulus intensity $\left(\sigma^{2}=a I^{n}\right.$, where $I$ is stimulus intensity), several major empirical laws of psychophysics can be derived from Equation 2, including Weber's law, Fechner's law, Stevens's law, and the magical number seven for channel capacity (Norwich, 1981, 1987). ${ }^{1}$

From Equations 3 and 4, it can be seen that in Norwich's informational approach, neural adaptation to a sensory stimulus is interpreted as the progressive acquisition of information from the stimulus as the stimulus persists, and thus the progressive decrease in uncertainty, or entropy, of the receptor-neuron unit with respect to that stimulus. A typical neural adaptation curve measured for a single auditory nerve (spiral ganglion) fiber in the guinea pig (characteristic frequency in the range $14-27 \mathrm{kHz}$ ) is shown in Figure 1 (Yates, Robertson, \& Johnstone, 1985). Notice that the rate of firing measured in the neuron decreased rapidly as time from the onset of the auditory stimulus increased, dropping from about 287 impulses per second shortly (about $2 \mathrm{msec}$ ) after stimulus onset to about 67 impulses per second at about $51 \mathrm{msec}$ after stimulus onset. This curve will be taken as a prototype of neural adaptation in the auditory receptor system, and will be compared in detail with psychophysical data in a later section of this paper. It should be noted that earlier adaptation curves that exhibited slower adaptation, such as those of Galambos and Davis (1943) and others since, were probably taken from more central fibers (see Galambos \& Davis, 1948; Yates et al., 1985). Apparently the very rapid adaptation discovered by Yates et al. (1985) is characteristic only of the primary receptor-neuron unit itself, which is, of course, the system to which Norwich's theory is supposed to apply.

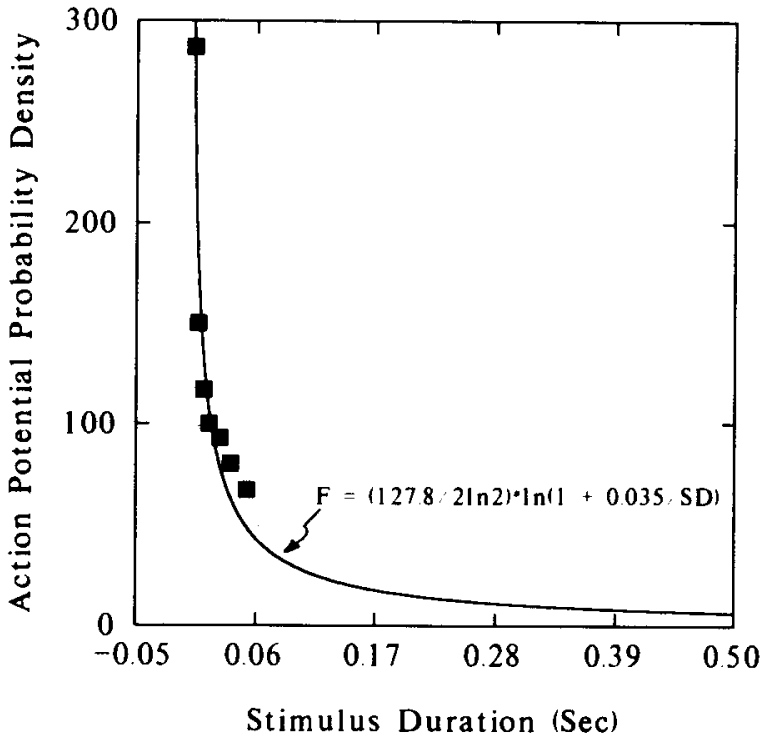

Figure 1. A typical neural adaptation curve. This one is for the response of a spiral ganglion cell in the guinea pig to a pure tone $12 \mathrm{~dB}$ above threshold. The cell had a characteristic frequency in the range 14-27 kHz; the specific frequency for this cell was not reported, since it was typical of all cells recorded. Points were read from a peristimulus histogram (Figure 5 in Yates, Robertson, \& Johnstone, 1985). Also shown is the best-fitting version of Equation $4(k=127.8, B=0.035)$.

According to Norwich's theory, the information acquired by the receptor-neuron unit sets a boundary condition on how much, and how fast, intensity information about a stimulus can be acquired by the whole organism. In Norwich's (1981) terms, the response of the receptorneuron unit calibrates the physical continuum so that its information content can be measured. For the receptorneuron unit, the shorter the duration of a stimulus, the less the available time within which to acquire information from it and the less the information that will be acquired. In fact, the adaptation curve can be viewed as an inevitable consequence of this, given the assumptions of the theory, a kind of conservation of information law. This law must, of course, be consistent with any explanation of adaptation that appeals to some mechanism, such as pre- or postsynaptic depletion of transmitter substance, and bears a relationship to such explanations that is similar to the relationship between the laws of thermodynamics and mechanistic theories in physics.

In order to apply the arguments based on the dynamics of single receptor-neuron units to the whole organism, Norwich (see especially Norwich, 1981) makes what turns out to be a critical assumption. He proposes a simple model in which all receptor-neuron units operate in parallel, "divested of their elaborate collateral connections. Thus, all receptors working together are taken to produce a simple amplification of the response of a single receptor" (Norwich, 1981, p. 414). Although this assumption would seem to entail several others about what 
happens more centrally where absolute identifications are made on the basis of the information so obtained, Norwich has not as yet discussed these assumptions in detail. The simplest, which was apparently intended, is that the information obtained by several independent channels is "averaged," providing decreased variability but no redundancy gain. In other words, if Channel 1 is "reporting" 1 bit of uncertainty reduction (or information transmitted) at time $t$ and Channel 2 is reporting 1.2 bits at the same time, the uncertainty reduction obtained by a higher center is taken to be 1.1 bits rather than 2.2 bits (as would be obtained, for example, if the higher center added the information obtained from the two channels). Because of the assumption of parallel noninteracting channels, the time course of information acquisition by a higher center would be roughly the same as that of any individual receptor-system unit (although it would be expected to be "smoother"); that is, the two time courses would be synchronous.

Since in this view the time course of information acquisition by the receptor-neuron unit limits the time course of information acquisition by the whole organism, we would expect that if the latter time course were measured psychophysically under appropriate conditions-for example, in the context of an absolute identification experiment-it would be synchronous with the neural adaptation curve. For short-duration stimuli, little information about stimulus intensity could be acquired, and uncertainty about the stimulus intensity would be large (information transmission would be low). The longer the stimulus persisted, the greater the amount of information about stimulus intensity that would be acquired and the less uncertainty there would be. By a careful analysis of neural adaptation curves, Norwich (1981) was able to calculate values for channel capacities (the maximum amount of information that can be transmitted by a sensory channel) in the range of those typically measured in psychophysical experiments (about 2-4 bits-see Garner, 1962; Miller, 1956). This lent credence to the postulated association between physiological adaptation of sensory receptorneuron units and psychophysically measured information acquisition by whole organisms.

Norwich (1981) suggested plotting stimulus equivocation (stimulus information minus information transmitted; see Garner \& Hake, 1951) from an absolute identification experiment versus stimulus duration to obtain an "informational adaptation curve" that could be compared to neural adaptation curves. ${ }^{2}$ Unfortunately, this definition of stimulus equivocation will not work for purposes of entropy theory, since stimulus information in an absolute identification experiment depends on the experimenter's (arbitrary) choice of how many stimuli to present for identification (stimulus information equals $\log _{2}$ of the number of equiprobable stimuli). It seems reasonable to take as the maximum stimulus equivocation (equivalent to stimulus information) the maximum amount of information that the whole organism could obtain from the stimulus-namely, the channel capacity. Therefore, in this paper, stimulus equivocation is defined as

$$
E_{s}=C-I_{t},
$$

where $E_{s}$ represents stimulus equivocation, $C$ represents channel capacity (defined for present purposes as the asymptotic information transmitted per stimulus in an absolute identification experiment as stimulus information is increased; see Garner, 1962), and $I_{t}$ represents information transmitted per stimulus. Norwich's entropy theory requires that stimulus equivocation $\left(E_{s}\right)$ and entropy $\left(H_{s}\right)$ be closely related. I assume the simplest relation

$$
E_{s}=H_{s} \text {, }
$$

where $H_{s}$ is as defined in Equation 3. Thus, we can write

$$
E_{s}=[1 /(2 \cdot \ln 2)] \cdot \ln (1+B / \tau) .
$$

Equation 7 represents an expression for the informational adaptation curve analogous to Equation 4 for the neural adaptation curve. Notice that the parameter $B$ is present in both equations. Since $B$ plays the role of the time constant in Equations 4 and 7, this amounts to the assertion that the time course of the informational adaptation curve should be synchronous with that of the adaptation curve of the receptor-neuron unit. I will report on a test of this property of the theory for the data to be reported in this paper.

In this paper, I report four experiments directed specifically at obtaining informational adaptation curves for auditory and visual intensity and comparing these curves with neural adaptation curves. A fifth experiment was addressed to a possible criticism of the other four, but in the auditory domain only. The experiments will be presented first in the order in which they were run, with descriptions of the reasoning that justified them. Then the relevant experimental results will be combined, to make the most meaningful possible comparison of informational and neural adaptation curves.

\section{EXPERIMENT 1}

Experiment 1 was designed to establish the range of information transmissions to be expected in this context and to get a preliminary estimate of the form of the auditory informational adaptation curve. To this end, large ranges of stimulus durations and intensities were used, and a large number of different stimulus intensities was used so that stimulus information would not limit information transmission. In spite of this intention, I neglected to obtain data at any stimulus durations less than $15 \mathrm{msec}$ in this and the subsequent two experiments. In these experiments, the shortest duration was chosen to be $15 \mathrm{msec}$ for a variety of reasons. First, this was the shortest duration for my equipment that met two very stringent conditions: (1) Rise and fall times must be long enough $(2.5 \mathrm{msec})$ so that enormous click transients were avoided; (2) the time during which signal amplitude was close to its nomi- 
nal amplitude must be very close to the nominal duration. For shorter duration signals, the effective duration of the effective signal is extended by the nonzero rise and fall times by an indefinite amount. Second, all previous studies typically had been done with somewhat longer durations (the shortest were 20,50 , or $100 \mathrm{msec}$, depending on the study), and $15 \mathrm{msec}$ was considered to be quite short in this context. Third, $10 \mathrm{msec}$ is the shortest duration for which a pure tone possesses the quality of being a "tone"; shorter duration tones sound like clicks. I thought that $15 \mathrm{msec}$ would be sufficient to make all tones clearly "tonal." Finally, the first three experiments were done before I discovered the Yates et al. (1985) paper, when 1 thought that the time course of adaptation of auditory nerve fibers was best exemplified by the Galambos and Davis (1943) results, for which both the 15- and the 45msec conditions should have given appreciably less information transmission than the longer duration conditions should. The Yates et al. (1985) paper made it clear that at least one empirical data point was needed below $15 \mathrm{msec}$. For this reason, a fourth experiment was run expressly to provide this point, although problems remain regarding just what that duration was (see Experiment 4). The experiment on visual intensity identification (Experiment 5) was also run subsequent to my discovery of the Yates et al. paper, and it included stimulus durations as short as $1 \mathrm{msec}$.

\section{Method}

Subjects. Three undergraduate students at the University of British Columbia were paid for their participation. None had any known hearing defects, and all could easily hear all of the stimuli. None had ever before participated in experiments of this sort.

Stimuli and Apparatus. The auditory stimuli were $1000-\mathrm{Hz}$ sinusoids generated, amplified, and electronically gated (2.5-msec rise and fall times) by a custom-built digitally controlled sound generator, and timed by a microcomputer (Intel 80286 processor), which also recorded subjects' responses and presented the feedback. The sounds were presented diotically through Koss Pro 4A earphones while subjects sat in a sound-attenuation chamber. Responses were made on a standard computer keyboard. Twenty-one different intensities (from 40 to $100 \mathrm{~dB}$ in 3-dB steps, measured at the earphone with a custom-built artificial ear and a General Radio precision sound-level meter) were presented equally often in Experiment 1. Since there were 21 different equiprobable intensities to be identified, available stimulus information was $\log _{2}(21)=4.39$ bits.

Procedure. Each subject made a 100 -trial practice run and then three 210-trial data runs in each of 6 stimulus-duration conditions $(15,45,135,405,1,215$, and $3,645 \mathrm{msec})$ in an absoluteidentification-with-feedback paradigm. The duration conditions were completed in a different irregular order for each subject. Each stimulus intensity was presented 10 times per data run in a different irregular order, for a total of 30 presentations per stimulus intensity per subject at each duration. Feedback, in the form of an illuminated numeral indicating the correct response, occurred immediately after the response was made to each stimulus. Information transmission was calculated from the resulting stimulus-response confusion matrices, using the method of Garner and Hake (1951).

\section{Results}

The data from Experiment 1 are displayed in Table 1. These data are the averages across subjects for the information transmission calculated from the confusion matrix
Table 1

Information Transmission Data from Experiments 1, 2, 4, and 5

\begin{tabular}{cccccc}
\hline Duration & Exp. 1 & Exp. 2 & Exp. 4 & Mean 1,2,4* & Exp. 5 \\
\hline 0.001 & & & & & 1.44 \\
0.005 & & & 1.08 & 1.61 & 1.43 \\
0.015 & 1.78 & 1.47 & 1.25 & 1.84 & 1.48 \\
0.045 & 1.87 & 1.77 & & 2.04 & 1.42 \\
0.135 & 1.90 & 1.67 & 1.42 & 2.01 & 1.54 \\
0.405 & 1.97 & 1.77 & & 2.09 & \\
1.215 & 1.94 & 1.83 & & 2.10 & \\
3.645 & 1.98 & & & 2.08 & \\
$t_{i}$ & 0.879 & 1.00 & 1.176 & & \\
\hline
\end{tabular}

*Mean of $\left(I_{t} \times t_{i}\right)+0.34$; see text.

for each subject in each duration condition on the basis of all 30 presentations of each stimulus-that is, each subject's three data runs in each duration condition were combined. (Stimulus equivocations, as defined by Equation 5, will be presented only for the combined data in a later section.) Information transmission was greater than zero for all conditions ( $p<.01$ by $t$ test). Remarkably, the average information transmission for the shortest duration condition $(15 \mathrm{msec})$ was 1.78 bits. Given that the maximum information transmission observed in this experiment was about 1.98 bits, it is clear that the majority of available information about sound intensity $190 \%$ in this experiment) is obtained in just $15 \mathrm{msec}$. This is consistent with the earlier work of Tulving and Lindsay (1967). A detailed comparison of the relationship between these data and the neural adaptation curve will be reported below under Comparison of Adaptation Curves.

What about the informational adaptation curve itself? Is further information obtained after the first $15 \mathrm{msec}$ ? In order to test the reliability of the effect of stimulus duration on information transmission, a repeated measures analysis of variance was done. Since sphericity was violated in these data, the (Huynh-Feldt) corrected degrees of freedom were used for the significance test. The effect of stimulus duration was not statistically significant at the .05 level $[F(4.5,9)=2.35, p=.13]$. Of course power is quite low for this test, because of the small number of subjects, because the relatively small number of presentations per stimulus (30) gives rise to a relatively large variance of the individual information transmissions, and because sphericity was violated.

\section{EXPERIMENT 2}

Experiment 2 was a replication of Experiment 1 (except for the longest stimulus duration) with fewer different stimulus intensities so that maximum information transmission could be optimally measured (information transmission can be lower than channel capacity when the number of stimuli and responses is large; see Garner, 1962). Power is greater in Experiment 2 because of a greater number of subjects and because variance was decreased by doubling the number of presentations of each stimulus. 


\section{Method}

Subjects. The subjects were 4 undergraduate students at the University of British Columbia who were paid for their participation. None had any known hearing defects, and none had ever before participated in an experiment of this sort.

Stimuli and Apparatus. The stimuli and apparatus were identical to those used in Experiment 1, except that only 11 different intensities were used: from 45 to $95 \mathrm{~dB}$, in 5-dB steps. Thus, the maximum stimulus information (maximum stimulus equivocation) was 3.46 bits.

Procedure. Each subject made a 100 -trial practice run and three 220-trial data runs in each of the five stimulus duration conditions $(15,45,135,405$, and $1,215 \mathrm{msec})$. Thus, each stimulus occurred 20 times in each run in a different irregular order, for a total of 60 presentations per stimulus per subject at each duration. Each subject completed the various duration conditions in a different irregular order.

\section{Results}

The data from Experiment 2 are displayed in Table 1. Clearly these data replicate the main features of those of Experiment 1. Again, information transmission was reliably different from zero in all conditions $(p<.01$ by $t$ test), and again most of the available information was obtained in the first $15 \mathrm{msec}$; information transmission averaged 1.47 bits for that stimulus duration, about $80 \%$ of the maximum of 1.83 bits in the 1,215 -msec condition. Sphericity was again violated, so that (Huynh-Feldt) corrected degrees of freedom were used to test significance in the analysis of variance for the effect of stimulus duration on information transmission. This effect was statistically significant $[F(4,12)=4.31, p=.02]$. Tukey tests $(\alpha=.05)$ showed that the 15 -msec condition was different from all but the 135-msec condition; no other differences were significant.

\section{EXPERIMENT 3}

Experiment 3 was run in order to counter the possible criticism that short auditory storage (Cowan, 1984), which can make information from an auditory stimulus available for up to $200 \mathrm{msec}$ or so following termination of that stimulus, was responsible for the high information transmission observed for the short stimulus durations (cf. Tulving \& Lindsay, 1967). The presentation of an intense mask immediately following the offset of the stimulus to be identified effectively terminates the short auditory store of that stimulus in detection and discrimination paradigms (see Cowan, 1984), although its effect is more problematic in absolute identification paradigms. Moreover, a similar procedure is used routinely to terminate sensory processing of visual stimuli (see, e.g., Haber \& Hershenson, 1980, pp. 136-138), and it is reasonable to expect that it would do the same for auditory stimuli, although to my knowledge it is not usually used in that way. Since the mask might also interfere with postpresentation cognitive processing of the stimulus itself in any of these paradigms, a decrease in information transmission under masking does not necessarily indicate that the short audi- tory store is extending the effective duration of the briefest stimuli when the mask is not present. However, substantial information transmission under such masking conditions would be evidence in favor of the view that information is obtained very rapidly by the auditory system. To explore this possibility, in Experiment 3, the 15-msec duration condition of the first two experiments was replicated under two conditions: stimuli presented alone, or stimuli presented and followed immediately by intense masking noise.

\section{Method}

Subjects. The subjects in Experiment 3 were the author and 2 graduate students at the University of British Columbia; all were experienced in psychophysical judgment tasks but had never done exactly this task before.

Stimuli and Apparatus. Everything was the same as in Experiments 1 and 2, except that only eight different stimulus intensities (from 50 to $92 \mathrm{~dB}$, in 6-dB steps) were used. The masking noise was a 500 -msec burst of broadband noise at $85 \mathrm{~dB}(\mathrm{C})$ presented immediately after the offset of the 15 -msec stimulus in the masking condition. This noise was judged effective in terminating the short auditory store for the 15 -msec stimulus without profoundly (backwardly) masking it, although some masking was expected.

Procedure. After from 1 to 3 practice runs, the subjects completed 480 trials (in runs of varying lengths) in each of the masked and unmasked versions of the 15-msec condition ( 2 did the masked condition first, 1 the unmasked condition), for a total of 60 judgments of each of the eight intensity levels in each condition.

\section{Results}

Information transmission for the unmasked condition (15-msec stimulus duration) averaged 1.72 bits in this experiment $(1.53,1.82$, and 1.80 for the 3 subjects individually); it averaged 1.07 bits in the masked condition (1.14, 0.92 , and 1.15). The value of 1.72 for the unmasked condition is somewhat larger than that in Experiments 1 and 2 (overall average, 1.57), possibly because all of the subjects in Experiment 3 were quite experienced. The value of 1.07 bits for the masked condition, although 0.65 bit lower than that for the unmasked condition $[t(2)=-4.39$, $p<.05$ ], is nevertheless substantially [and reliably, $t(2)=14.26, p<.01]$, larger than zero. Also, some masking of postpresentation cognitive processing may have taken place, since such masks have been shown to interfere with several different aspects of absolute identification performance (Mori \& Ward, 1990). Thus, it is implausible that the substantial performance observed in the 15-msec condition in the first two experiments arose merely from an extension of the effective duration of the stimulus by the short auditory store. It seems that it is possible to extract most of the intensity information from an auditory stimulus in only $15 \mathrm{msec}$.

\section{EXPERIMENT 4}

Experiment 4 was run after I became aware of the paper by Yates et al. (1985). That paper makes it clear that much of the action in the neural adaptation curve occurs before 
$15 \mathrm{msec}$ after stimulus onset. Although suggestive, the data from Experiments 1-3 are deficient in that they do not measure stimulus equivocation for durations shorter than $15 \mathrm{msec}$. In fact, the data from Experiments 1 and 2 are generally quite commensurate with the data of Yates et al. for durations equal to or greater than $15 \mathrm{msec}$. As will be seen (see below under Comparison of Adaptation Curves), this is misleading. Experiment 4 was run expressly to provide another data point in the region below $15 \mathrm{msec}$, at about $5 \mathrm{msec}$ (nominal duration). In addition, an attempt was made to minimize the amount of "onset splatter" of the stimuli by limiting the maximum intensity.

\section{Method}

Subjects. The subjects were 4 experienced students and 1 inexperienced student at the University of British Columbia. One of the experienced subjects had participated in Experiment 3. The others were new to this series of experiments. All had normal hearing at $1000 \mathrm{~Hz}$, and all could detect all of the stimuli used.

Stimuli and Apparatus. The apparatus was the same as that used in Experiments 1-3. The stimuli were six $1000-\mathrm{Hz}$ sinusoids, ranging in amplitude from 40 to $65 \mathrm{~dB}$ in 5-dB steps (stimulus information $=2.585$ bits). Fewer and softer stimulus intensities were used in order to decrease the influence of onset transients, because these are larger the more intense the stimulus. The nominal stimulus durations were 5,15 , and $135 \mathrm{msec}$. The effective durations were approximately the same as the nominal durations, except possibly in the 5-msec condition. Since the rise and fall times were each $2.5 \mathrm{msec}$, it is possible that some extension of the effective stimulus to, say, 6 or $7 \mathrm{msec}$ occurred. Nonetheless, this was the shortest duration at which the faintest of the stimuli could be detected by all of the subjects, and so it was used. The stimulus and duration sets were thus the result of a compromise between the desire to investigate very short durations and the desire to limit onset transients that might contribute to identifiability.

Procedure. Since the objective in this study was to obtain asymptotic performance, especially in the 5-msec condition, a bit of liberty was taken with the order in which conditions were run. All subjects initially completed one session in each of the three duration conditions in one of three irregular orders. Each session consisted of 100 practice trials followed by 360 data trials $(60$ per stimulus intensity). However, the shorter conditions proved to be difficult for the subjects who completed them first. In the 5-msec condition, the stimuli appeared to be very faint clicks and were easy to miss altogether. Therefore, 2 subjects completed an additional session in the 5-msec condition, and 1 subject completed two additional sessions in the 5-msec condition and one in the 15-msec condition, by which times it was judged that their performances had stabilized. Only the data of the final session for each condition for each subject are reported here.

\section{Results}

The average information transmissions obtained in this experiment are displayed in Table 1. Information transmissions in the 15- and 135 -msec conditions were somewhat lower than in Experiments 1 and 2, as might be expected from the more difficult stimulus ensemble used in Experiment 4 . The value observed in the 5-msec condition, however, was still an appreciable fraction (76.1\%) of that in the $135-\mathrm{msec}$ condition, indicating that a great deal of the available intensity information was obtained in only about $5 \mathrm{msec}$.

\section{EXPERIMENT 5}

I ran Experiment 5 because I thought it was possible that onset splatter could still be artifactually increasing the amount of intensity information available for the shortest duration sounds in Experiment 4. It seemed necessary to test the theory on a continuum in which better control of very short duration stimuli was possible. I chose to use light intensity. Although the neural adaptation data available are not as satisfactory as those for audition, they suggest a somewhat slower time course for adaptation of retinal ganglion cells. Transient cells have been found to decrease their firing rate to background levels within $0.5 \mathrm{sec}$ after onset of a visual stimulus (Cleland \& EnrothCugell, 1968), although they can be slower than this (Cleland, Dubin, \& Levick, 1971); sustained cells decrease firing rate to a level substantially above background over a period of up to $15 \mathrm{sec}$ (Cleland et al., 1971). Thus, a combination of well-controlled stimuli and a relatively slow time course for physiological adaptation should yield a fairly definitive test of the prediction that the informational and neural adaptation curves will be synchronous.

\section{Method}

Subjects. Three experienced University of British Columbia students participated for pay. Two had participated in one or the other of Experiments 1-4, but none were aware that the present experiment was in any way related to the earlier ones. All had normal or corrected-to-normal vision, as measured with a Snellen test.

Stimuli and Apparatus. The stimuli were approximately point sources (1-mm disk at $80-110 \mathrm{~cm}$ from the eye) with luminances of $1.030,2.318,5.214,11.73,26.40$, and $59.39 \mathrm{fL}$. These were the weakest stimuli that met the requirements that the smallest intensity must be reliably detectable in the $1-$ msec condition and that information transmission be substantial (at least 1.5 bits) in the 135 msec condition. The stimuli were generated by an LED that emitted photons with a wavelength of approximately $565 \mathrm{~nm}(1 / 2$ peak bandwidth about $50 \mathrm{~nm}$ ). The rise and fall times for the LED were less than $100 \mu \mathrm{sec}$. The stimuli were presented and timed, and data recorded, with a Hewlett-Packard Vectra ES/12 microcomputer, as in the earlier experiments. The subjects fixated the light source for each stimulus presentation by centering their gaze with respect to a square array of four dim red LEDs mounted $3.2 \mathrm{~cm}$ from the center LED, but head position was not rigidly controlled or monitored. It is supposed that presentation was foveal. The subjects were seated in a sound-attenuation chamber that was illuminated only by a dim red light over the keyboard upon which responses were made.

Procedure. The subjects were dark-adapted for $2 \mathrm{~min}$ before beginning their judgments. Each session consisted of 50 practice trials followed by 360 data trials ( 60 per stimulus intensity). Two subjects participated in one session each in each of the five duration conditions $(1,5,15,45$, and $135 \mathrm{msec})$. One subject repeated the 5 -msec condition (his first) after all conditions had been completed once, because it was clear that his performance was not at asymptote in the first session in that condition. Only the data from his second run in the 5-msec condition are reported here.

\section{Results}

The average results from this experiment are displayed in Table 1, calculated in the same way as for the previous experiments. Overall performance is in the range of 
other light-intensity identification experiments but somewhat lower than the nominal channel capacity of about 2 bits (Garner, 1962; Miller, 1956). Since conditions in the present experiment were designed to assess changes in information transmission with changes in stimulus duration, and not to maximize information transmission, this is not surprising. Nonetheless, performance in the longer duration conditions is good enough (about 1.5 bits) so that the effects of duration would be easily discernible, if present. In fact, however, they are not present. Information transmission for $1-\mathrm{msec}$ stimuli is only 0.1 bit less than that for 135-msec stimuli. Acquisition of visual intensity information is very rapid indeed-if anything, even more rapid than for auditory intensity.

Again, it is possible that some kind of short visual store, or perhaps visual persistence (see, e.g., Di Lollo, 1977), extended the effective duration of the shorter stimuli so that all effective durations were at least $100 \mathrm{msec}$. It is difficult to deal with this potential criticism, except to say that because the stimuli were all very faint indeed, such persistence would be expected to be minimal. Moreover, a masking study like that in Experiment 3 could have been done for the visual stimuli, but it is unclear what could be learned. It would be difficult to create conditions in which faint 1-msec stimuli could be detected in the presence of an immediately subsequent intense mask, given the energy summation that occurs in the visual system.

\section{COMPARISON OF ADAPTATION CURVES}

The fifth column of Table 1 (labeled "Mean 1,2,4") displays a sort of maximum likelihood estimate of the informational adaptation curve for sound intensity. It was obtained by multiplying the average information transmissions at each duration in each of Experiments 1, 2, and 4 by a factor that made average information transmission for the 135-msec condition (shared by Experiments 1, 2, and 4) equal to 1.67, the mean for that condition in Experiment 2 . The average across experiments for each duration condition was then taken. Finally, 0.34 was added to the overall average (transformed) values to make the information transmission at $\mathbf{1 . 2 1 5} \mathrm{sec}$ equal to the channel capacity for auditory intensity (about 2.1 bits; Garner, 1962; Miller, 1956).

Table 2 presents the stimulus equivocations (channel capacity minus information transmitted) for the combined (transformed) data of Table 1. It also presents in numerical form the data of Yates et al. (1985) that were plotted in Figure 1, and the results of fitting the equations of entropy theory to both data sets. Notice that the first data point for the combined auditory data represents maximum stimulus equivocation (channel capacity) and is indicated to occur at $1 \mathrm{msec}$. This implements the arbitrary, although reasonable, assumption that at the moment at which the stimulus is first detected ( $1 \mathrm{msec}$ ?), information about it is minimal (information transmitted so far $=0$ bits). The first data point in the Yates et al. (1985) data, at 2 msec, represents the first histogram bar in the
Table 2

Auditory Data and Results of Curve Fitting

\begin{tabular}{|c|c|c|c|c|c|c|}
\hline \multicolumn{3}{|c|}{ Guinea pig ganglion cell } & \multicolumn{4}{|c|}{ Human psychophysical data } \\
\hline$\tau$ & PDF & $F_{\text {pred }}$ & $\tau$ & $E_{\mathrm{s}}$ & & ed \\
\hline .002 & 287 & 270 & .001 & 2.10 & 1.80 & 2.58 \\
\hline .006 & 150 & 178 & .005 & 0.49 & 0.84 & 1.50 \\
\hline .011 & 117 & 132 & .015 & 0.26 & 0.40 & 0.87 \\
\hline .016 & 100 & 107 & .045 & 0.06 & 0.16 & 0.42 \\
\hline .026 & 93 & 79 & .135 & 0.09 & 0.06 & 0.17 \\
\hline .036 & 80 & 63 & .405 & 0.01 & 0.02 & 0.06 \\
\hline .051 & 67 & 48 & & & & \\
\hline$B$ & & 0.035 & & & 0.011 & 0.035 \\
\hline SS $_{\text {res }}$ & & $2,194.01$ & & & 0.245 & 1.760 \\
\hline
\end{tabular}

Note $-\tau=$ stimulus duration; PDF $=$ probability density function from Yates et al. (1985); $F_{\text {pred }}=$ predicted firing rate from Equation 4; $E_{\mathrm{s}}=$ stimulus equivocation using data from Experiments 1, 2, and 4; $B=$ parameter value from Equations 4 and $7 ; S_{\text {res }}=$ sum of squared residuals from fits of Equations 4 and 7 .

plot of probability density versus duration presented in their paper. This makes the excursions of the curves in the two data sets roughly commensurable and allows the data of both data sets to be used in the comparison procedure that follows.

In order to compare fits of Equations 4 and 7 to the appropriate data, I excluded the data points for stimulus durations of 1.215 and $3.465 \mathrm{sec}$ for the psychophysical data in order that the longer durations (during which stimulus equivocation was changing very little) not dominate the parameter values obtained. This seems reasonable, since it is the early time courses that we wish to compare. In making this restriction, the fits for the psychophysical data are rendered less stable, but this is compensated for by more meaningful fits.

The data of Yates et al. (1985) that are displayed in Figure 1 and Table 2 were chosen for comparison to the data from the present auditory experiments. These data are single representative points read from a peristimulus histogram (1- to 2-msec bins) of the action potential probability density (roughly equivalent to firing rate) from a spiral ganglion cell exposed repeatedly (total of 500 exposures) to a pure tone of $100-\mathrm{msec}$ duration $12 \mathrm{~dB}$ above threshold at the cell's characteristic frequency (Figure 5 in Yates et al.). This cell was chosen by Yates et al. as a typical one; they reported very little variability in the rapid adaptation part of such cells' responses to stimuli. The time course of the very rapid adaptation did change somewhat with stimulus intensity, however, being faster for higher intensity stimuli. The data in Figure 1 represent the middle of the range of time courses found by Yates et al.

Yates et al. (1985) fitted a fairly complicated two-stage (faster and slower adaptation) negative exponential model to their data. Power functions of time with negative exponents also fit such data well. However, for present purposes it was considered sufficient to fit Equation 4 to the neural firing data and Equation 7 to the psychophysical data. The curve fitting was carried out by using a simplex optimization algorithm (Nelder \& Mead, 1965) implemented in a QuickBASIC computer program (modi- 
fied from one kindly supplied by $K$. Norwich) run on an Intel 80386-based microcomputer with an 80387 arithmetic coprocessor (Hewlett-Packard Vectra QS/20). The program used the least squares error criterion and the fits reported are comparable to those of other equations (e.g., power functions) with a similar number of parameters.

Table 2 juxtaposes the numerical values of the neural data fitted and the values "predicted" by the best-fitting version of Equation 4, which had the parameter values $k=127.8$ and $B=0.035$. The fitted equation is also drawn in Figure 1. It can be seen from Figure 1 and from Table 2 that the fit to the neural firing data is respectable. It looks about as good as the fits of the models suggested by Yates et al. (1985), which were, however, based on assumptions about the actual physiological processes involved in neural adaptation. Thus, both equations derived from entropy theory, based on assumptions of conservation of information, and equations capturing various process assumptions provide reasonable accounts of the adaptation curves of peripheral auditory receptor-neuron units.

Table 2 also juxtaposes the combined psychophysical data, providing an overall estimate of the auditoryintensity informational adaptation curve, with predictions made by inserting the time constant parameter, $B=$ 0.035 , into Equation 7. If the neural and informational adaptation curves are synchronous, this equation should provide an acceptable description of the informational curve. The informational adaptation curve and the predictions of Equation 7 are plotted in Figure 2. It is clear that Equation 7 with $B=0.035$ is not a good description of

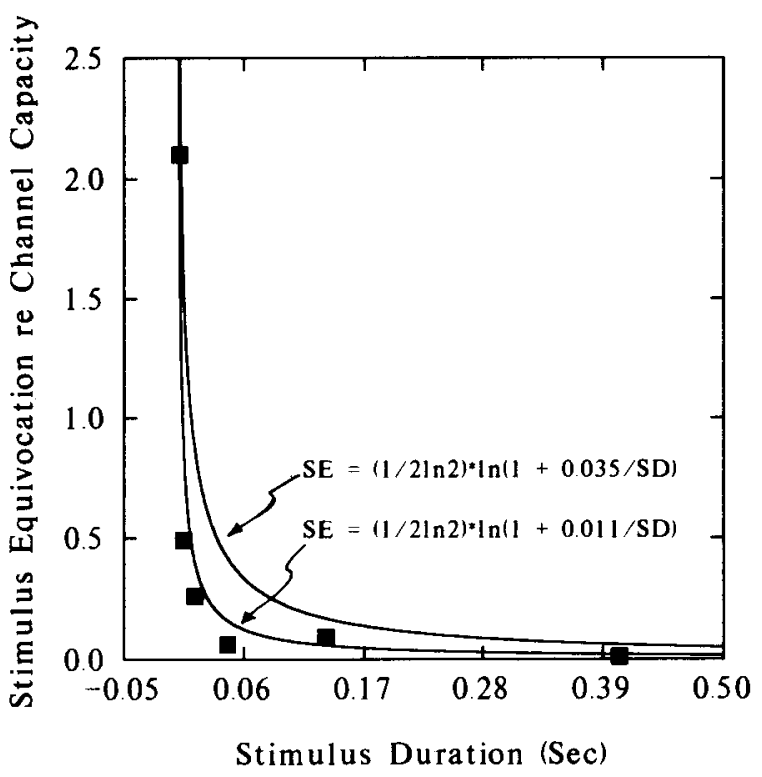

Figure 2. The informational adaptation curve for auditory absolute identification. Data plotted are transformed mean stimulus equivocations (Experiments 1, 2, and 4) from Table 2. Also shown are the version of Equation 7 having the same $B$ value $(0.035)$ as that in Equation 4 in Figure 1, and the best-fitting version of Equation $7(B=0.011)$.
Table 3

Visual Data and Results of Curve Fitting

\begin{tabular}{|c|c|c|c|c|c|c|}
\hline \multicolumn{3}{|c|}{$\begin{array}{c}\text { Cat Retinal Ganglion } \\
\text { Cell }\end{array}$} & \multicolumn{4}{|c|}{ Human Psychophysical Data } \\
\hline \multirow{2}{*}{$\begin{array}{c}\tau \\
.023\end{array}$} & \multirow{2}{*}{$\begin{array}{c}F \\
271\end{array}$} & \multirow{2}{*}{$\begin{array}{c}F_{\text {pred }} \\
274\end{array}$} & \multirow{2}{*}{$\begin{array}{c}\tau \\
.00005\end{array}$} & \multirow{2}{*}{$\begin{array}{c}E E_{\mathrm{s}} \\
2.00\end{array}$} & \multicolumn{2}{|c|}{$E_{s}$ pred } \\
\hline & & & & & 1.90 & 5.60 \\
\hline .038 & 224 & 213 & .001 & 0.10 & 0.36 & 3.45 \\
\hline .067 & 153 & 153 & .005 & 0.11 & 0.09 & 2.31 \\
\hline .096 & 94 & 121 & .015 & 0.06 & 0.03 & 1.57 \\
\hline .126 & 106 & 100 & .045 & 0.12 & 0.01 & 0.93 \\
\hline .155 & 85 & 85 & .135 & 0.00 & 0.00 & 0.45 \\
\hline 185 & 76 & 74 & & & & \\
\hline .214 & 76 & 66 & & & & \\
\hline 244 & 66 & 59 & & & & \\
\hline 274 & 59 & 54 & & & & \\
\hline 302 & 48 & 50 & & & & \\
\hline .332 & 38 & 46 & & & & \\
\hline 361 & 44 & 43 & & & & \\
\hline 391 & 44 & 40 & & & & \\
\hline .420 & 38 & 37 & & & & \\
\hline $\boldsymbol{B}$ & & 0.118 & & & 0.00064 & 0.118 \\
\hline $\mathbf{S S}_{\text {res }}$ & & 1143.25 & & & 0.091 & 0.760 \\
\hline
\end{tabular}

Note $-\tau=$ stimulus duration; $F=$ firing rate from Cleland and EnrothCugell (1968); $F_{\text {pred }}=$ predicted firing rate from Equation $4 ; E_{\mathrm{s}}=$ stimulus equivocation using data from Experiment $5 ; B=$ parameter value from Equations $\mathbf{4}$ and $7 ; \mathbf{S S}_{\text {Tes }}=$ sum of squared residuals from curve fits.

the psychophysical data. Table 2 and Figure 2 also contain the predictions made from the best-fitting equation of the form of Equation 7, which had $B=0.011$. This value of $B$ clearly provides a better fit (the residual error is nearly an order of magnitude smaller) to the psychophysical data, indicating that the time course of acquisition of information by the whole organism (decrease of stimulus equivocation with time) is substantially faster than (asynchronous with) that of single receptor-neuron units (represented by decline of firing rate with time).

What about the visual data? As I mentioned earlier, the time course of adaptation to constant-intensity stimuli (small spots of light) of retinal ganglion cells is somewhat slower than that of spiral ganglion cells to sounds. Firing in at least some central retinal (foveal) ganglion cells declines to background rate over a time period of at least $0.5 \mathrm{sec}$ (Cleland \& Enroth-Cugell, 1968). Table 3 and Figure 3 present the results of fitting Equation 4 to data from a single, representative cat retinal ganglion cell of this type read from a graph presented by Cleland and Enroth-Cugell. The exact instant at which the stimulus was applied is not determinable from the graph, so the time scale has been adjusted to give the best curve fit. The resulting curve fit is quite good, with parameter values of $k=210$ and $B=0.118$, and it is comparable to the fit of Equation 4 to the Yates et al. (1985) data. Notice that the larger value of $B$ reflects the slower time course of neural adaptation for the retinal ganglion cell. These fits can be contrasted with those to the psychophysical data (transformed to equivocations re a channel capacity of 2.0). For these curves it was assumed that equivocation was 2.0 at a duration of $0.00005 \mathrm{sec}$, because this value of duration gave the best fits. Table 3 
and Figure 4 present these equivocations and the "predictions" of Equation 7 with $B=0.118$. The predictions of the best-fitting version of Equation 7 ( $B=0.00064$, sum of squared residuals $=0.091$ ) are also included there. Again, the version of Equation 7 using the $B$ estimated from the neural data does worse at predicting the equivocations than does the best-fitting version, which has a much smaller $B$. Clearly the time courses for the neural and the informational curves are asynchronous for visual intensity.

Now we can use Norwich's (1981) method to estimate channel capacity for the neural adaptation curves:

$$
C=\left(F_{\max }-F_{\min }\right) / k,
$$

where $C$ is estimated channel capacity, $F_{\max }$ and $F_{\min }$ are firing rates at stimulus onset and after complete adaptation, respectively, and $k$ is estimated from Equation 4. Estimating $F_{\max }$ to be about 300 and $F_{\min }$ to be about 40 for the auditory data in Figure 1 and their analysis by Yates et al. (1985), we obtain a channel capacity of roughly $(300-40) / 127.8=2.03$ bits for sound intensity, very close to the value of 2.1 reported by Garner (1962) and Miller (1956) and assumed here for the calculation of stimulus equivocation. Similarly, for the retinal ganglion cell reported by Cleland and Enroth-Cugell (1968), $F_{\max }=$ about $274, F_{\min }=$ about 0 , and $k=210$, so that channel capacity is predicted to be about $(274-0) / 210=$ 1.3. This is somewhat smaller than the maximum value of roughly 2.0 bits usually quoted for light intensity (see, e.g., Garner, 1962); but it is close to the maximum measured in the present experiment (1.54 bits), and, given

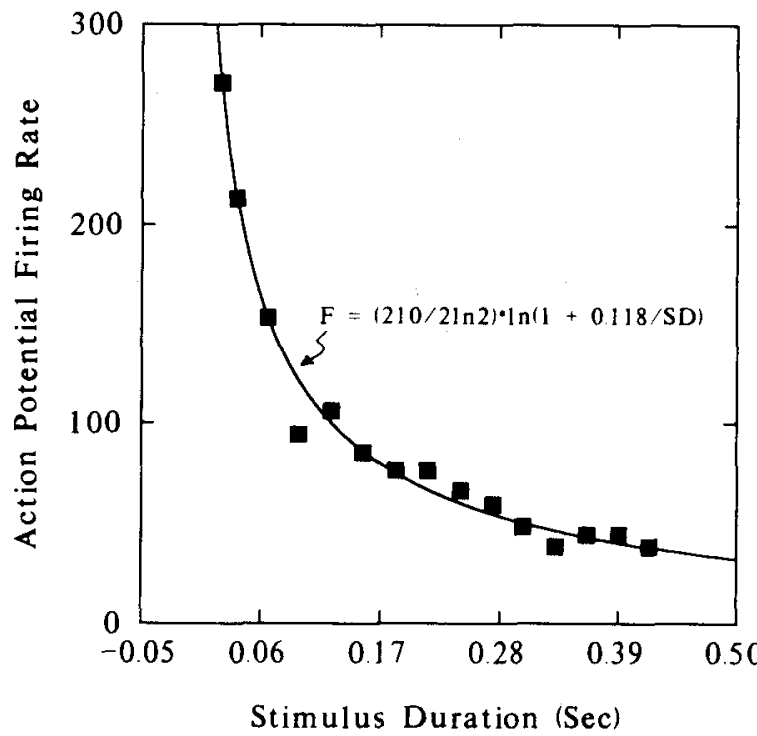

Figure 3. The neural adaptation curve for the response of a cat central fovea retinal ganglion cell to a $0.13^{\circ}$ spot of light of $1.3 \mathrm{log} \mathrm{Td}$ intensity on a background of $0 \log \mathrm{Td}$. Average firing rates for 60 presentations of a square-wave stimulus were read from Figure 2 of Cleland and Enroth-Cugell (1968). Also shown is the best-fitting version of Equation $4(k=210, B=0.118)$. Digitization and curve fit from Norwich (personal communication).

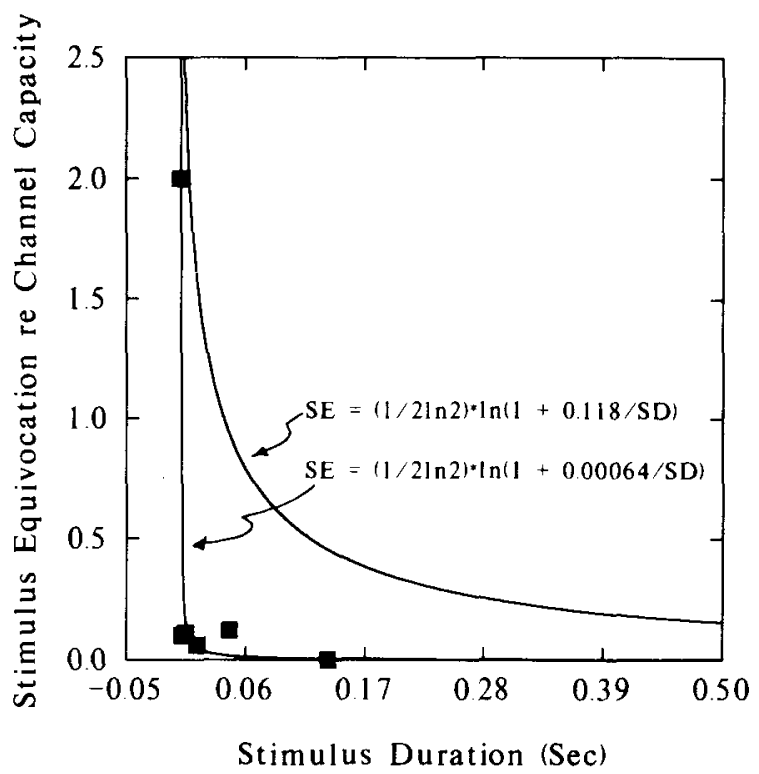

Figure 4. The informational adaptation curve for visual absolute identification. Data plotted are mean stimulus equivocations (Experiment 5) from Table 3. Also shown are the version of Equation 7 having the same $B$ value $(0.118)$ as that in Equation 4 in Figure 3 , and the best-fitting version of Equation $7(B=0.00064)$.

the uncertainty inherent in reading precise data from published graphs, it is sufficient to allow the conclusion that the total amount of information mediated by the neural and informational adaptation curves is roughly the same.

The above data make clear that stimulus duration has surprisingly little effect on information transmission of suprathreshold stimuli over a range of a few milliseconds to several seconds. This is because information transmission for even the shortest duration stimuli is a large proportion ( $>75 \%)$ of that available. This is unexpected, both because of traditional dogma and because of the numerous other situations in which stimulus duration has major effects, such as absolute and differential threshold measurements and identification of complex patterns.

It is also unexpected from the simplest possible interpretation of Norwich's entropy theory of sensory systems. Making the assumption that all neural channels convey information in parallel, or at best average their entropies at points of convergence, informational and neural adaptation curves are expected to be synchronous. It is clear that although the informational and neural adaptation curves do resemble each other in overall form, the rate of information acquisition is substantially greater for the whole organism than it is for an individual nerve fiberthat is, the curves are asynchronous. I believe that the most plausible explanation for this is that the whole organism (or some subsystem of it) sums the information provided by the several available nerve fibers transmitting information about any stimulus.

One way to see this is in the context of Norwich's (e.g., 1981) ideas about what is going on at the level of the individual receptor-neuron unit. As outlined in the introduc- 
tion, each receptor is supposed to sample the stimulus (viewed as a flux of quantized energy bundles) at some rate. As the samples accumulate, the variability of the sampling distribution of the mean of the samples decreases. The entropy of the receptor-neuron unit, being a monotone increasing function of this variability, also decreases as samples accumulate, and, on the assumption that neural firing is proportional to entropy, the neural adaptation curve is the result. Consider the situation for stimuli whose duration $(t)$ is less than the interval between two samples. In the simple model proposed by Norwich (1981), in which nerve fibers act in parallel and redundantly, the number of samples would be 1 or less and the amount of reduction in receptor entropy (information transmitted) would be very small. However, consider a system in which several (say $N$ ) of the nerve fibers converged on a higher order neuron. If each of the converging fibers were to be able to take only one sample of the stimulus, the higher order neuron would have available information from $N$ samples. It would thus act, for this short-duration stimulus, as if it were a single nerve fiber sampling the stimulus over a duration $N t$, instead of over the actual duration, $t$, of the stimulus. If identification judgments were based on the activity of this higher order neuron, there would be little effect of stimulus duration on information transmission if all stimuli were of a duration sufficient to yield at least one sample of the stimulus from a sufficiently large number of receptors. This would seem to be the case, at least for suprathreshold auditory and visual stimuli. As long as there is enough stimulus energy available to trigger responses in several usually quiescent receptors, convergence of these receptors on a higher order neuron will allow the integration of the entropy reductions experienced by the individual receptors into a larger entropy reduction at the site of convergence. This would hold regardless of modality, since such convergence is a feature of all known sensory systems.

Why would such convergence be important in identification tasks but not in threshold tasks, where stimulus duration does have dramatic effects (e.g., Bloch's law, which holds for audition as well as vision; see Scharf \& Buus, 1986)? In the case of very faint stimuli, the problem is to obtain even one sample, since very few quantized energy bundles are available to the receptors. For shortduration near-threshold stimuli, convergence of $N$ receptors on a second order neuron would increase the probability of obtaining one sample, but it is unlikely that all or even several of the receptors would receive a quantum of energy. The longer the duration of the stimulus, the more the quanta that will arrive in the vicinity of the receptors. If perception of the presence of a stimulus depends on the reception of a fixed number of quanta (e.g., as in Hecht et al., 1942), longer duration stimuli will reach threshold at lower intensities (fluxes) (up to the limit of temporal integration, about $100 \mathrm{msec}$ ). Similarly, the problem in differential threshold measurements is to discriminate the samples of one stimulus from those of another. Any factor that would affect the variability of a sampling distribution would affect discriminability of two such distributions, and duration would do this, even if $N$ samples were available in each time interval. Thus, this approach indicates how stimulus duration could have major effects on absolute and differential thresholds but little effect on absolute identification of suprathreshold stimuli, at least for durations of greater than $1 \mathrm{msec}$.

The convergence approach just outlined would predict that any stimulus of long enough duration to allow one sample to be taken by each of several receptors would yield about the same information transmission. The fact that more central neural responses to 1 -msec "click" stimuli change with stimulus intensity similarly to the way responses to longer duration pure tones do (see, e.g., Gulick, 1971) is also consistent with the convergence view.

The idea that neural convergence mediates information accumulation over time is similar to an assumption typically made in mechanistic models of information accumulation, such as counting models in which the random variable on which identification, detection, or discrimination decisions are based is a count of the number of neural impulses that occur in a fixed interval of time (see Luce, 1986). In a representative model of this type, the stimulus is supposed to affect $J$ channels for a duration $\delta$. For simplicity's sake, it is assumed that counts are made from a single channel for a time $J \delta$. It can be shown that a measure of discrimination, $d^{\prime}$, can be written as a function of the observation time, $\delta$, which is usually taken to be the stimulus duration:

$$
d^{\prime}=a(J \delta)^{1 / 2},
$$

where $a$ is a constant that is a function of the intensities of the stimuli involved and the variability of their sensory effects across trials (see Luce, 1986). Such counting models clearly account for the effect of stimulus duration on discrimination. This model can be extended to absolute identification by using, for example, a model like the very successful one of Braida and Durlach (1988), in which the index of identification is the sum of the $d^{\prime}$ values for all adjacent pairs of stimuli in the ensemble to be identified. Since $a$ depends on the stimulus intensities, we could write an extended model as follows:

$$
\sum d^{\prime}=\sum a_{i}(J \delta)^{1 / 2}=(J \delta)^{1 / 2} \sum a_{i} .
$$

This type of model predicts a similar duration effect for discrimination and for identification. Since the effect was not found for identification, the model must be incomplete, but it is not immediately clear how.

\section{CONCLUSIONS}

The results reported in this paper have several implications for the applicability to psychophysics of a theory like Norwich's, which emphasizes conservation laws and boundary conditions rather than mechanistic models of processes. A deeper understanding of the process of information acquisition by an organism, and of the effects 
of stimulus duration on that process, was attained through the comparison of adaptation curves via the theoretical equations. Norwich's (1981) suggestion that both the informational and the neural adaptation curves represent, or mediate, the same total quantity of information, transmitted by peripheral receptor-neuron units to the whole organism, was confirmed within the limits of the availability of appropriate neural data. However, his suggestion that informational adaptation curves would be synchronous with neural adaptation curves, derived from a sort of minimal version of the theory as applied to the whole organism, was not confirmed. The two types of adaptation curve appear to be asynchronous. Some mechanistic understanding of the process of information acquisition by the whole organism via peripheral and central neural systems, or of how information available to sensory systems is used in making absolute identifications, would probably be required to advance our understanding of this asynchrony. And although the speculations about neural convergence, combined with the assumption that identification decisions are made after such convergence occurs, do account for the results obtained, they remain speculations. If confirmation of predictions made from such a revision of Norwich's theory is obtained, such confirmation would indicate that a "conservational" understanding of information acquisition is possible. Such an understanding places limits on mechanistic explanations but does not inform them directly. Eventually, mechanistic and conservational explanations of all available data must converge in order for psychophysics to become more complete.

\section{REFERENCES}

Braida, L. D., Durlach, N. I. (1988). Peripheral and central factors in intensity perception. In G. M. Edelman, W. E. Gall, \& W. M. Cowan (Eds.), Auditory function (pp. 559-583). New York: Wiley.

Brondegnt, D. E. (1958). Perception \& communication. London: Pergamon.

Cleland, B. G., Dubin, M. W., \& Levick, W. R. (1971). Sustained and transient neurones in the cat's retina and lateral geniculate nucleus. Journal of Physiology, 217, 473-496.

Cleland, B. G., E Enroth-Cugell, C. (1968). Quantitative aspects of sensitivity and summation in the cat retina. Journal of Physiology, 198, 17-38.

Cowan, N. (1984). On short and long auditory stores. Psychological Review, 96, 341-370.

Di LoLLo, V. (1977). Temporal characteristics of iconic memory. $\mathrm{Na}$ ture, 267, 241-243.

Egeth, H., \& PAChella, R. (1969). Multidimensional stimulus identification. Perception \& Psychophysics, 5, 341-346.

Florentine, M. (1986). Level discrimination of tones as a function of duration. Joumal of the Acoustical Society of America, 79, 792-798.

GalAmBos, R., DAvis, H. (1943). The response of single auditorynerve fibers to acoustic stimulation. Jourmal of Neurophysiology, 6 , 39-57.

Galambos, R., \& Davis, H. (1948). Action potentials from single auditory nerve fibers? Science, $108,513$.

GARNER, W. R. (1962). Uncertainty and structure as psychological concepts. New York: Wiley.

GaRner, W. R., C Creelman, C. D. (1964). Effect of redundancy and duration on absolute judgments of visual stimuli. Journal of Experimental Psychology, 67, 168-172.

GARNER, W. R., \& HAKE, H. (1951). The amount of information in absolute judgments. Psychological Review, 58, 446-459.

GuLICK, W. L. (1971). Hearing: Physiology and psychophysics. New York: Oxford University Press.

HABER, R. N., \& HERSHENSON, M. (1980). The psychology of visual perception. New York: Holt, Rinehart \& Winston.

Hecht, S., Schlaer, S., \& Pirenne, M. H. (1942). Energy, quanta, and vision. Journal of General Physiology, 25, 819-840.

Hughes, J. W. (1946). The threshold of audition for short periods of stimulation. Proceedings of the Royal Society of London: Series B, 133, 486-490.

LOCKHEAD, G. R. (1966). Effects of dimensional redundancy on visual discrimination. Joumal of Experimental Psychology, 72, 95-104.

LUCE, R. D. (1986). Response times: Their role in inferring elementary mental organization. New York: Oxford University Press.

Miller, G. A. (1956). The magical number seven, plus or minus two: Some limits on our capacity for processing information. Psychological Review, 63, 81-97.

MoRI, S. (1989). A limited-capacity response process in absolute identification. Perception \& Psychophysics, 46, 167-173.

MORI, S., \& WARD, L. M. (1990). Unmasking the magnitude estimation response. Canadian Journal of Psychology, 44, 58-68

Nelder, J. A., MEAD, R. (1965). A simplex method for function minimization. Computer Joumal, 7, 308-313.

NoRWICH, K. H. (1977). On the information received by sensory receptors. Bulletin of Mathematical Biology, 39, 453-461.

NorWICH, K. H. (1981). The magical number seven: Making a "bit" of "sense." Perception \& Psychophysics, 29, 409-422.

Norwich, K. H. (1987). On the theory of Weber fractions. Perception \& Psychophysics, 42, 286-298.

Paquet, L., \& Merikle, P. M. (1984). Global precedence: The effect of exposure duration. Canadian Journal of Psychology, 38, 45-53.

Scharf, B., BuUs, S. (1986). Audition I. In K. Boff, L. Kaufman, \& J. Thomas (Eds.), Handbook of perception and performance (pp. 14-1 to 14-71). New York: Wiley.

Shannon, C. E. A. (1948). A mathematical theory of communication. Bell System Technical Journal, 27, 379-423, 623-656.

Tolman, R. C. (1938). The principles of statistical mechanics. London: Oxford University Press.

TULVING, E., \& LiNDSAY, P. H. (1967). Identification of simultaneously presented simple visual and auditory stimuli. Acta Psychologica, 27, 101-109.

Yates, G., Robertson, D., \& Johnstone, B. M. (1985). Very rapid adaptation in the guinea pig auditory nerve. Hearing Research, 17, $1 \cdot 12$.

\section{NOTES}

1. Substituting $\sigma^{2}=a I^{n}$ into Equation 4 and letting $B^{\prime}=a / \gamma \tau N^{2}$, we obtain

$$
F=[k /(2 \cdot \ln 2)] \cdot \ln \left(1+B^{\prime} I^{n}\right) .
$$

This shows that, in the entropy theory, receptor-neuron unit firing rate, $F$, is a function of stimulus intensity for stimuli of constant duration, which is consistent with the traditional interpretation of receptor firing rate as encoding stimulus intensity (although this encoding may be changed and/or interpreted at more central sites).

2. Norwich (1981) actually used the term "psychophysical adaptation curve." I have changed this to "informational adaptation curve," which is more precise and is not confusable with other forms of adaptation curves measured psychophysically, such as adaptation in the magnitudes of sensations (loudness adaptation) or changes in sensitivity of sensory systems (darkness adaptation).

(Manuscript received July 17, 1989; revision accepted for publication March 1, 1991.) 